International Journal of Current Microbiology and Applied Sciences

ISSN: 2319-7706 Volume 10 Number 11 (2021)

Journal homepage: http://www.ijcmas.com

\title{
Detection of Carbapenemase Production in Clinical Isolates of Escherichia coli and Klebsiella Species
}

\author{
Harvinder Kaur*, Sneha Mohan, Tarana Sarwat and Dalip K. Kakru \\ Department of Microbiology, School of Medical Science \& Research (SMS\&R), Sharda \\ University, Greater Noida, India \\ *Corresponding author
}

\section{Keywords}

Enterobacteriaceae, CarbapenemResistant Enterobacteriaceae, Carbapenemase

\section{Article Info}

Received: 10 October 2021 Accepted: 02 November 2021 Available Online: 10 November 2021

\section{A B S T R A C T}

The increasingly frequent application of carbapenemases induces a selective pressure on bacteria to acquire resistance against carbapenems. A large variety of carbapenemases have been identified in Enterobacteriaceae. The emergence of carbapenemases in E. coli and Klebsiella species possess a serious therapeutic problem in hospitals because carbapenems are often antibiotics of last resort for the treatment of serious infections caused by multidrug-resistant bacteria. The main aim of this study to determine the carbapenem-resistant strains of Escherichia coli and Klebsiella species. Detection of carbapenemase enzyme in the carbapenem-resistant Escherichia coli and Klebsiella species. This is a crosssectional study, performed in the bacteriological laboratory. A total of 204 strains of Escherichia coli and 122 strains of Klebsiella species were isolated from patients admitted \& attending the OPD at Sharda Hospital during the study period. The identification of the clinical isolates was based on morphological and biochemicals characterization. Result: Among the total number of bacterial growth received during the study period, the E. coli (204) was more in number as compared to other bacterial strains followed by Klebsiella species (122), Pseudomonas species (117), Staphylococcus aureus (100) and Enterococcus species (80). Among the following specimen i.e, urine (58.8\%), was highly received during the study period followed by pus (15.3\%) and sputum (7.5\%). Colistin (100\%) and Polymyxin B (100\%) is found to be the most sensitive drug. Gentamicin (19.6\%) and Cefuroxime (12.2\%) are the least sensitive drugs. In the present study, we found that the Klebsiella species (75\%) and Escherichia coli (64\%) was the major reason of carbapenemase production but the organisms incidentally were susceptible to colistin and polymyxins (100\%) followed by other antibiotics. This is a welcome finding in times of increasing carbapenem resistance $\&$ is reason enough to suggest the use of Polymyxins in the health care settings where the carbapenems to be used for the treatment of infections caused by Enterobacteriaceae. 


\section{Introduction}

The genus Escherichia and Klebsiella belong to the family of Enterobacteriaceae. Escherichia coli are one of the most common members of the family Enterobacteriaceae and often exist as commensal in the gastrointestinal tract of humans and animals.

Klebsiella pneumoniae is the most clinically relevant Klebsiella species and is responsible for over $70 \%$ of human infections due to this genus ${ }^{(1)}$. In humans, $K$. pneumoniae most often colonizes the gastrointestinal tract, skin, and nasopharynx and is an important cause of serious community-onset infections such as necrotizing pneumonia, pyogenic liver abscesses, and endogenous endophthalmitis ${ }^{(2)}$.

Various types of infections are caused by $E$. coli and Klebsiella species including urinary tract infections, neonatal meningitis, skin infections, sepsis, and intestinal infections more frequently than other members of the Enterobacteriaceae.

The increasingly frequent application of carbapenemases induces a selective pressure on bacteria to acquire resistance against carbapenems. A large variety of carbapenemases have been identified in Enterobacteriaceae belonging to three classes of beta-lactamases: classes A, B, and D ${ }^{(4)}$. These classes are of the greatest clinical importance among nosocomial pathogens.

The emergence of carbapenemases in E. coli and Klebsiella species possess a serious therapeutic problem in hospitals because carbapenems are often antibiotics of last resort for the treatment of serious infections caused by multidrug-resistant bacteria. These bacteria have the potential to spread rapidly within the hospital environment and also across the continent $^{(6)}$. Detection of carbapenemases thus plays a crucial role as an infection control issue because they are often associated with extensive antibiotic-resistant, treatment failures and infections ${ }^{(7) .}$

The present study is conducted to identify the carbapenemase resistance in E. coli and Klebsiella species from both in and outpatient samples in our tertiary care hospital.

The main aim of this study includes to determine the carbapenem-resistant strains of Escherichia coli and Klebsiella species. Detection of carbapenemase enzyme in the carbapenem-resistant Escherichia coli and Klebsiella species.

\section{Materials and Methods}

The study was conducted in a bacteriology laboratory in Sharda Hospital, Greater Noida. It was a cross-sectional study. The study was conducted from December-2019 to November 2020. All the clinical isolates of Escherichia coli (E. coli) \& Klebsiella species are processed during the study period.

All the carbapenem-resistant E. coli and Klebsiella species isolates were included in the study. Whereas exclusion criteria were not applicable in the study.

Methodology including all the specific specimens, tests for species identification such as direct microscopy (gram stain), hanging drop, biochemical reactions (catalase, oxidase, ICUT, MR test, VP test, nitrate reduction test, oxidation-fermentation test, decarboxylase test, sugar fermentation test, and AST.

\section{Incubation of culture media}

For incubation Bacteriological incubator was used which helps to grow and maintain the bacteriological culture at $37^{\circ} \mathrm{C} .{ }^{(8)}$ 


\section{Sample Processing}

All the isolates of clinical samples were processed for E. coli \& Klebsiella species as per the standard guidelines (9).

Antimicrobial Susceptibility Testing was performed on Mueller-Hinton Agar (MHA) by Kirby-Bauer Disk-Diffusion method as the screening was done by this method as per the CLSI guidelines 2019 (9).

Carbapenem resistance was detected by interpreting the zone diameters of imipenem, meropenem, and ertapenem as per the CLSI 2019 guidelines (9).

Detection of carbapenemase in carbapebem resistant E. coli and Klebsiella species.

\section{Screening Test}

Screening tests in the laboratory, acarbapenem-intermediateor- the resistant result should always raise the suspicion of possible carbapenemase production, as they should reduce carbapenem susceptibility within the susceptible range in isolates of members of the family Enterobacteriaceae. The best carbapenem for screening is unknown. In the present study, the screening was done by the Kirby Bauer Disk-Diffusion method.

\section{Disk diffusion method (Kirby Bauer)}

The Kirby-Bauer test, also known as the disk diffusion method, is the most widely used antibiotic susceptibility test in determining what choice of antibiotics should be used when treating an infection. ${ }^{(15)}$

\section{Confirmatory test}

A confirmatory test in the laboratory was done to confirm the resistance of the carbapenems which was screened during the screening method.

Ethylenediaminetetraacetic acid (EDTA) impregnated disc test

An enhancement in the zone of inhibition in the area between the Imipenem alone and the EDTA-Imipenem combined discs in comparison with the zone of inhibition on the far side of the drug was interpreted.

\section{Observations and Results}

A total no. of 204 strains of Escherichia coli and 122 strains of Klebsiella species were isolated from patients admitted \& attending the OPD at Sharda Hospital during the study period (1st December 2019 - 30th November 2020).

The table given below depicts the number of patient samples received in the bacteriology laboratory for culture \& sensitivity during the study period.

Among the total number of bacterial growth received during the study period, the $E$. coli (204) was more in number as compared to other bacterial strains followed by Klebsiella species (122), Pseudomonas species (117), Staphylococcus aureus (100), and Enterococcus species (80).

\section{Demographic Distribution of Various Samples Collected}

Out of 204 isolated organisms, $48 \%$ were from male patients and $51.9 \%$ were from female patients indicating higher rates of samples collected from females during a study period.

Out of 122 isolated organisms, $38.8 \%$ were from female patients and $61.4 \%$ from male patients indicating higher rates of samples collected from males during a study period 


\section{Age-Wise Distribution of Organisms}

The maximum number of isolates were obtained from the age group (21-30) i.e, (25.4\%) in Escherichia coli and $(22.1 \%)$ in Klebsiella species followed by the age group (41-50 years) of age i.e, (14.7\%) and (15.5\%) and followed by (61-70years) i.e, (9.8\%) and (20.4\%) in Escherichia coli and Klebsiella species respectively.

Among the following specimen i.e, urine $(58.8 \%)$, was highly received during the study period followed by pus $(15.3 \%)$ and sputum $(7.5 \%)$ as shown in the previous table 4 .

\section{Antibiotic Susceptibility Pattern of Enterobacteriaceae in Escherichia Coli and Klebsiella Species}

\section{Escherichia Coli}

There are 4 classes of antibiotics tested for antibiotics susceptibility in Enterobacteriaceae. These included -lactams (penicillins, cephalosporins, and carbapenems), aminoglycosides (gentamicin), fosfomycin, fluoroquinolones (levofloxacin, ciprofloxacin), tetracyclines, and polymyxins (colistin and Polymyxin B).

Amongst all the classes, polymyxins emerged as the most effective class of drugs against $E$. coli followed by Fosfomycin and penicillins.

\section{Klebsiella Species}

Amongst all the antibiotics, polymyxins (colistin and polymyxin B) were found to be most effective against Klebsiella species. They were followed by Fosfomycin, Tetracycline, Meropenem, Imipenem, and Ertapenem. Whereas, Gentamicin and Amoxyclav were found to be the most resistant drugs. Also, Cefuroxime was found to show resistance.

\section{Screening Test}

Out of 326 clinical isolates of GNB, 139(42\%) isolates were resistant to carbapenems (imipenem, ertapenem, meropenem).

A total number of 64 isolates of Escherichia coli were carbapenem-resistant from 204 isolates. Whereas, a total number of 75 isolates of Klebsiella species were carbapenem-resistant from 122isolates.

A total number of 139 isolates were carbapenem-resistant in Escherichia coli and Klebsiella species out of 326 isolates.

Prevalence of MBL production was highest in Klebsiella species $(51.8 \%)$, followed by Escherichia coli (48.1\%), as shown in table 8.

All the isolates were identified based on culture characteristics, gram staining, and biochemical reactions. The isolates were subjected to antimicrobial susceptibility testing.

Out of 326 clinical isolates of GNB, 133(42\%) isolates were resistant to carbapenems (imipenem, ertapenem, meropenem).

Prevalence of MBL production was highest in Klebsiella species $(51.8 \%)$, followed by Escherichia coli (48.1\%), as shown in the previous table.9.

\section{Results and Discussion}

The present study was conducted to know the prevalence of carbapenemase production in clinical isolates of Escherichia coli and Klebsiella species in our hospital and to know their antibiotic susceptibility profiles and resistance patterns. In our study 868 bacterial isolates were cultured from various $(13,639)$ clinical specimens over 12 months, 
$204(23.5 \%)$ isolates were identified as Escherichia coli and 122(14\%) as Klebsiella species.

Higher prevalence was observed in a study conducted by André Birgy et al., in 2012 where E. coli was $57.7 \%$. in another study conducted by Wonkeun Song, et al., in 2015, $23.8 \%$ Klebsiella species were isolated(20).

In this study, the maximum number of isolates were obtained from the age group (21- 30 years) i.e, $(25.4 \%)$ in Escherichia coli and $(22.1 \%)$ in Klebsiella species followed by the age group (41-50 years) of age i.e, (14.7\%) and $(15.5 \%)$ and followed by (61- 70years) i.e, (9.8\%) and (20.4\%) in Escherichia coli and Klebsiella species respectively. The high prevalence was observed in E. coli (33\%) and low prevalence was observed in Klebsiella species (13\%) in a study done by Kathleen Chiotos et al., in 2016.(19) Bacteriological profile in our study revealed the maximum number of strains of Escherichia coli and Klebsiella species in this study were isolated from urine $(58.8 \%)$, followed by pus $(15.3 \%)$ and sputum $(7.5 \%)$ to be the most common carbapenem-resistant pathogens. This result was comparable with the study done by Setegn Eshetie et al., in 2015, maximum isolated Enterobacteriaceae were from urine $(87.6 \% \%)$.

Amongst all the strains of Klebsiella species (122), the commonest species isolated in human clinical specimens was Klebsiella pneumoniae 106 (86.8\%) followed by Klebsiella oxytoca 16(13.1\%). This was similar to the study done by Sakina Yousuf Ali, et al., in 2020. ${ }^{(23)}$

Table.1 Disk diffusion method (Kirby Bauer)

\begin{tabular}{|c|c|c|c|}
\hline Screening drugs & Dose & $\begin{array}{c}\text { Zone Diameter (mm) } \\
\text { Sensitive(S) }\end{array}$ & Resistant (R) \\
\hline Ertapenem & $10 \mu \mathrm{g}$ & $\geq 22$ & $\leq \mathbf{1 8}$ \\
\hline Imipenem & $10 \mu \mathrm{g}$ & $\geq 23$ & $\leq \mathbf{1 9}$ \\
\hline Meropenem & $\mathbf{1 0 \mu \mathrm { g }}$ & $\geq \mathbf{2 3}$ & $\leq \mathbf{1 9}$ \\
\hline
\end{tabular}

Table.2 Total samples received during the Study Period

\begin{tabular}{|c|c|c|}
\hline \multicolumn{3}{|c|}{ IPD } \\
\hline Positives & Negatives & Total \\
\hline $\mathbf{N}(\%)$ & $\mathbf{N}(\%)$ & \\
\hline $\mathbf{7 6 2}(\mathbf{2 0 . 6 \%})$ & $\mathbf{2 9 3 0}(\mathbf{7 9 . 3 \%})$ & $\mathbf{3 6 9 2}$ \\
\hline \multicolumn{3}{|c|}{ OPD } \\
\hline $\begin{array}{c}\text { Positives } \\
\mathbf{N}(\%)\end{array}$ & $\begin{array}{c}\text { Negatives } \\
\mathbf{N}(\%)\end{array}$ & Total \\
\hline $497(4.9 \%)$ & $\mathbf{9 4 5 0}(\mathbf{9 5 \%})$ & $\mathbf{9 9 4 7}$ \\
\hline & Total Samples \\
\hline 1259 & $\mathbf{1 2 3 8 0}$ & $\mathbf{1 3 6 3 9}$ \\
\hline
\end{tabular}


Table.3 Organisms received during the study period.

\begin{tabular}{|c|c|}
\hline IPD & 762 \\
\hline OPD & 497 \\
\hline Escherichia coli & 204 \\
\hline Klebsiella species & 122 \\
\hline Pseudomonas species & 117 \\
\hline Staphylococcus aureus & 100 \\
\hline Enterococcus species & $\mathbf{8 0}$ \\
\hline
\end{tabular}

Table.4 Age-Wise Distribution of Organisms

\begin{tabular}{|c|c|c|}
\hline Age Group & Escherichia coli (\%) & Klebsiella species (\%) \\
\hline$<1 D$ to $10 y$ & 10.7 & 9.8 \\
\hline 11 to $20 y$ & 6.3 & 3.27 \\
\hline 21 to $30 y$ & 25.4 & 22.1 \\
\hline 31 to $40 y$ & 14.7 & 9.8 \\
\hline 41 to $50 y$ & 14.7 & 15.5 \\
\hline 51 to $60 \mathrm{y}$ & 8.8 & 8.1 \\
\hline 61 to $70 y$ & 9.8 & 20.4 \\
\hline 71 to $80 y$ & 11.27 & 09 \\
\hline 81 to $90 \mathrm{y}$ & 0.98 & 1.6 \\
\hline 90 to $100 y$ & - & 0.8 \\
\hline
\end{tabular}

Table.5 Specimen Wise Distribution of Escherichia Coli and Klebsiella Species

\begin{tabular}{|c|c|c|c|}
\hline Specimen & $\begin{array}{c}\text { Escherichia coli } \\
(\mathbf{n}=\mathbf{2 0 4})\end{array}$ & $\begin{array}{c}\text { Klebsiella species } \\
(\boldsymbol{n}=\mathbf{1 2 2})\end{array}$ & Total $(\mathbf{n}=\mathbf{3 2 6}) /(\%)$ \\
\hline Urine & 153 & 39 & $\mathbf{1 9 2}(\mathbf{5 8 . 8})$ \\
\hline Pus & 27 & 22 & $\mathbf{4 9}(\mathbf{1 5 . 3 \%})$ \\
\hline Sputum & 07 & 16 & $\mathbf{2 3}(\mathbf{7 . 5 \%})$ \\
\hline Blood & 04 & 09 & $\mathbf{1 3}(\mathbf{3 . 9 \%})$ \\
\hline Stool & 06 & 0 & $\mathbf{0 6}(\mathbf{1 . 8 \%})$ \\
\hline Tip, (catheter, ETT) & - & 22 & $\mathbf{2 2}(\mathbf{6 . 7 \%})$ \\
\hline $\begin{array}{c}\text { Bronchio alveolar } \\
\text { lavage (BAL) }\end{array}$ & 02 & 02 & $\mathbf{0 4}(\mathbf{1 . 2 \%})$ \\
\hline Body Fluids & 01 & 01 & $\mathbf{0 2}(\mathbf{0 . 6 \%})$ \\
\hline Swabs & - & 01 & $\mathbf{0 1}(\mathbf{0 . 3 \%})$ \\
\hline Tracheal Secretion & - & 07 & $\mathbf{0 7}(\mathbf{2 . 1 \%})$ \\
\hline TISSUE & 01 & 02 & $\mathbf{0 3}(\mathbf{0 . 9 \%})$ \\
\hline Bronchial Aspirate & 01 & 01 & $\mathbf{0 2}(\mathbf{0 . 6 \%})$ \\
\hline Bile & $\mathbf{0 1}$ & - & $\mathbf{0 1}(\mathbf{0 . 3 \%})$ \\
\hline
\end{tabular}


Table.6 Distribution of carbapenem-resistant isolates

\begin{tabular}{|c|c|c|}
\hline Total number of isolates & $\mathbf{3 2 6}$ & $\mathbf{2 5 . 8 \%}$ \\
\hline Carbapenem-resistant in $\boldsymbol{E}$. coli & 64 & $\mathbf{4 6 \%}$ \\
\hline Carbapenem-resistant in Klebsiella species & 75 & $\mathbf{5 3 . 9 \%}$ \\
\hline Total Carbapenem-Resistant isolates & $\mathbf{1 3 9}$ & $\mathbf{4 2 . 6 \%}$ \\
\hline
\end{tabular}

Table.7 Showing Screening Positives for Escherichia coli and Klebsiella species

\begin{tabular}{|c|c|c|}
\hline & $\begin{array}{c}\text { Escherichia coli } \\
n=64\end{array}$ & Klebsiella species $\boldsymbol{n}=\mathbf{7 5}$ \\
\hline Screening Positives & IPM + EDTA & IPM + EDTA \\
\hline $\mathbf{n = 1 3 9}$ & 40 & 44 \\
\hline Percentage $(\%)$ & 62.5 & 58.6 \\
\hline
\end{tabular}

Table.8 Showing Prevalence of MBL Production

\begin{tabular}{|c|c|c|c|}
\hline & Escherichia coli & Klebsiella species & Total \\
\hline ISOLATES & 204 & 122 & $\mathbf{3 2 6}$ \\
\hline MBL (Total) MBL & 64 & 75 & $\mathbf{1 3 9}$ \\
(Positives) MBL & 08 & 27 & $\mathbf{3 5}$ \\
(Weakly Positives) & & & $\mathbf{4 9}$ \\
\hline PERCENTAGE (\%) & 32 & 17 & $\mathbf{4 0 . 7 \%}$ \\
\hline
\end{tabular}

Fig.1

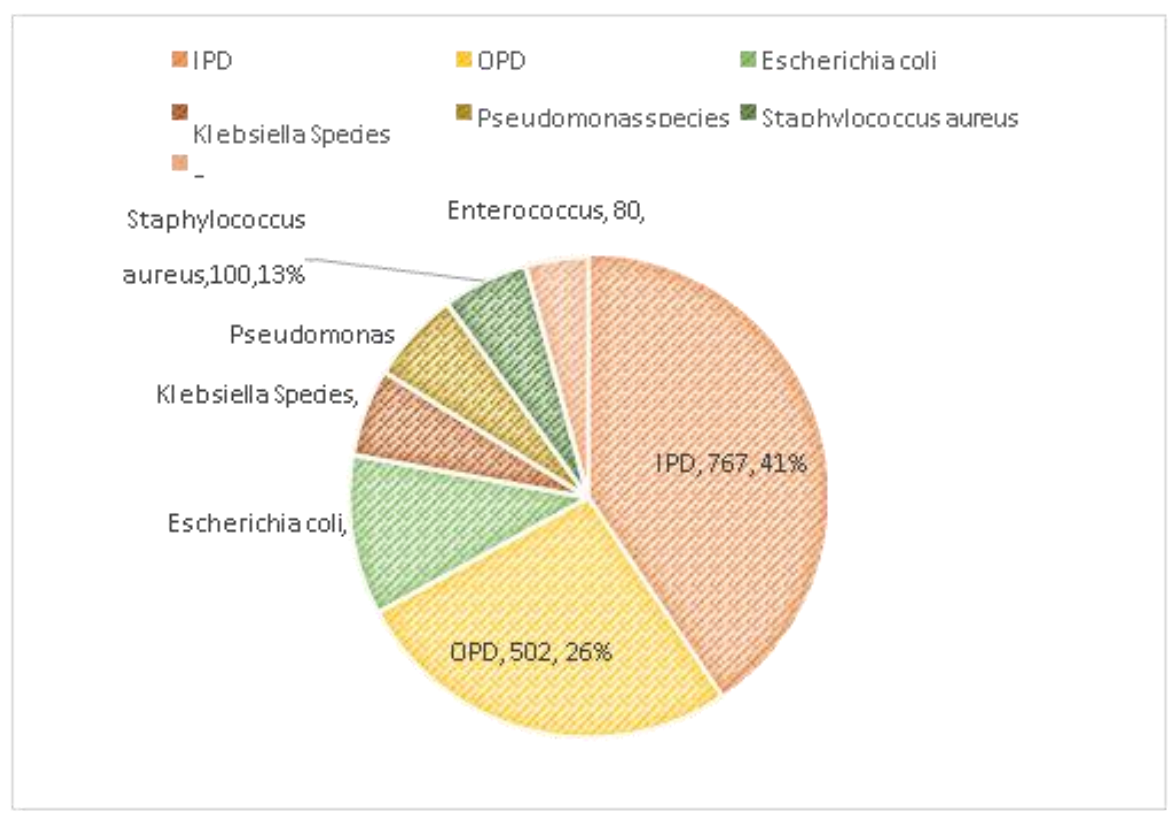


Fig.2 Showing Male: Female Ratio for Escherichia coli.

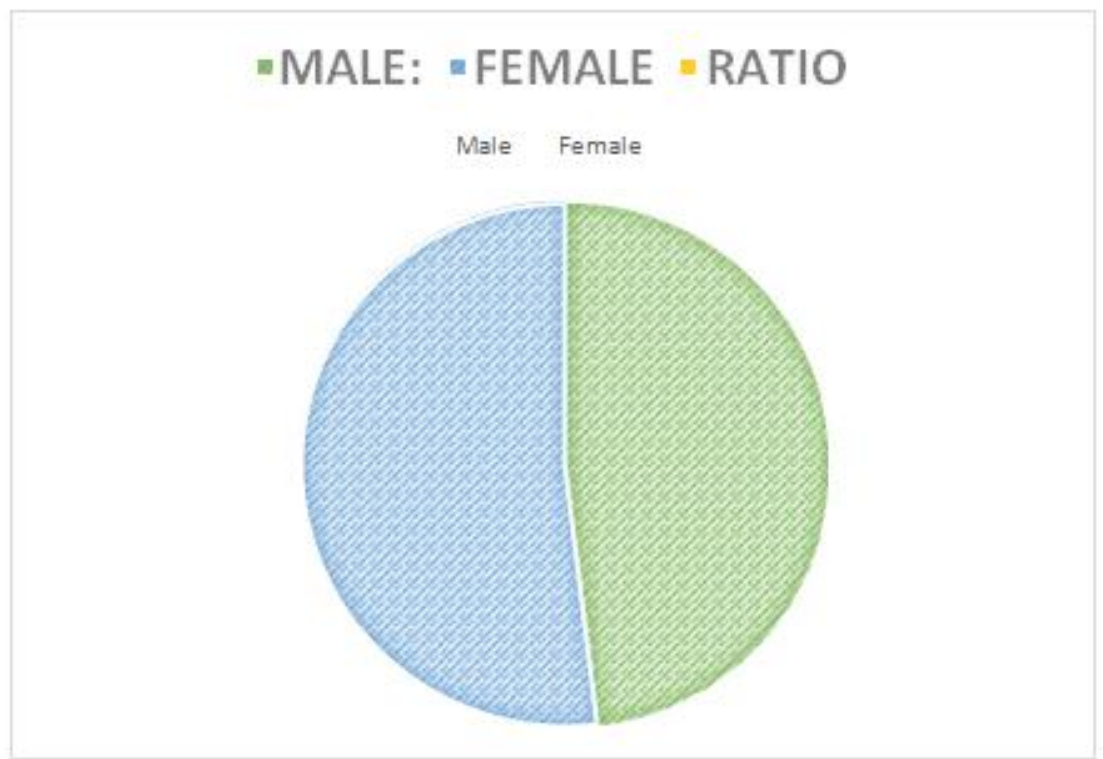

Fig.3 Showing Male: Female Ratio for Klebsiella species.

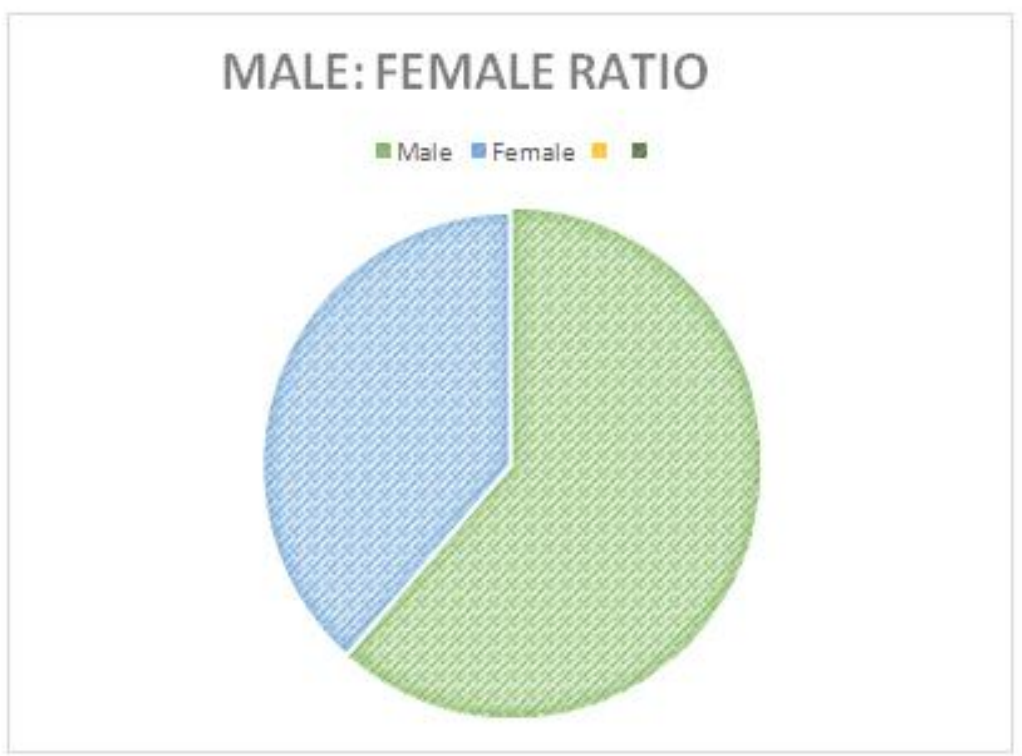


Fig.4

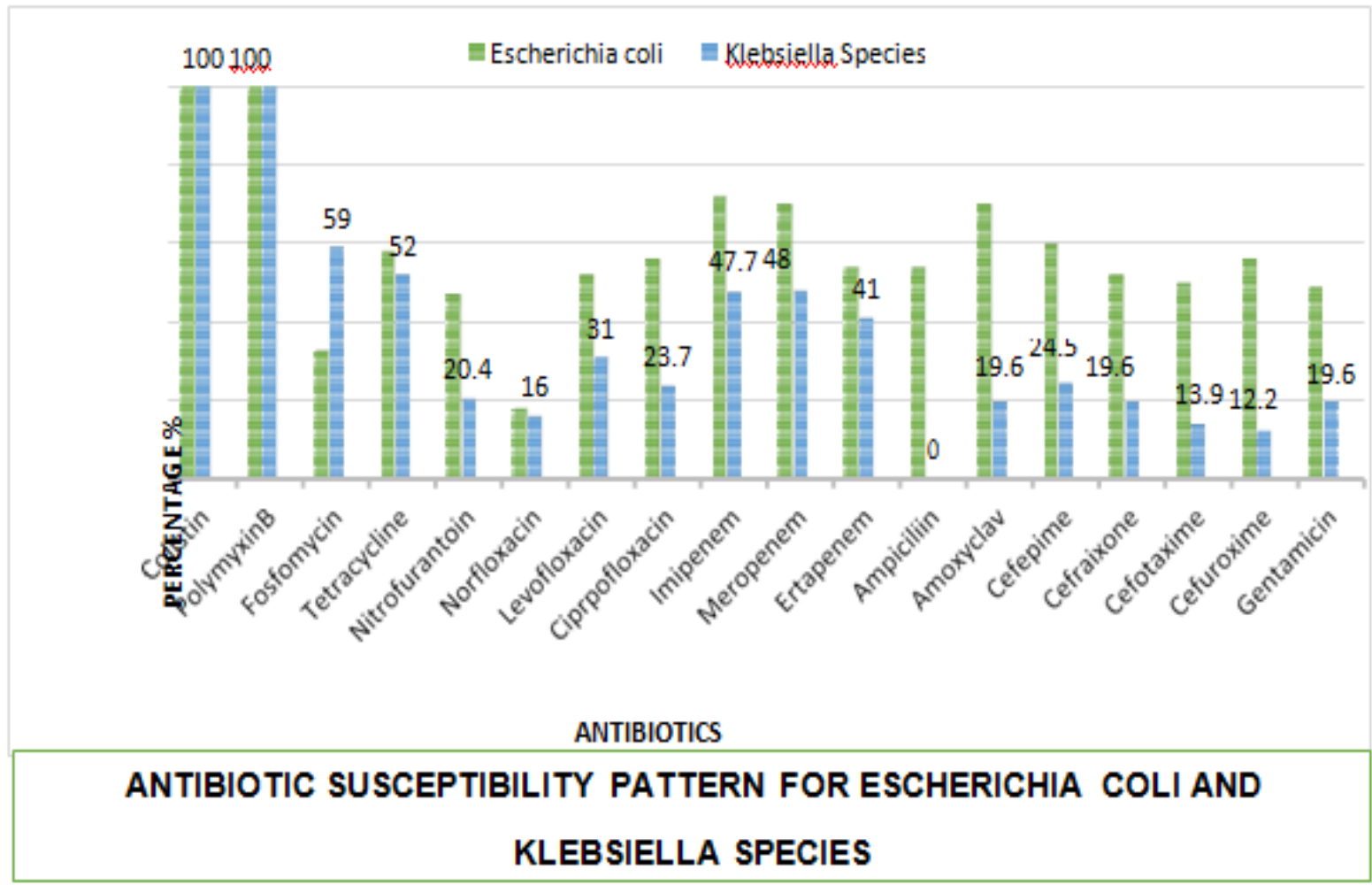

Colistin (100\%) and Polymyxin B (100\%) is found to be the most sensitive drug. Gentamicin (19.6\%) and Cefuroxime $(\mathbf{1 2 . 2 \%})$ are the least sensitive drugs.

Fig.5 Screening Test

\section{POSITIVE SAMPLES}

MBLproducers Total isolates

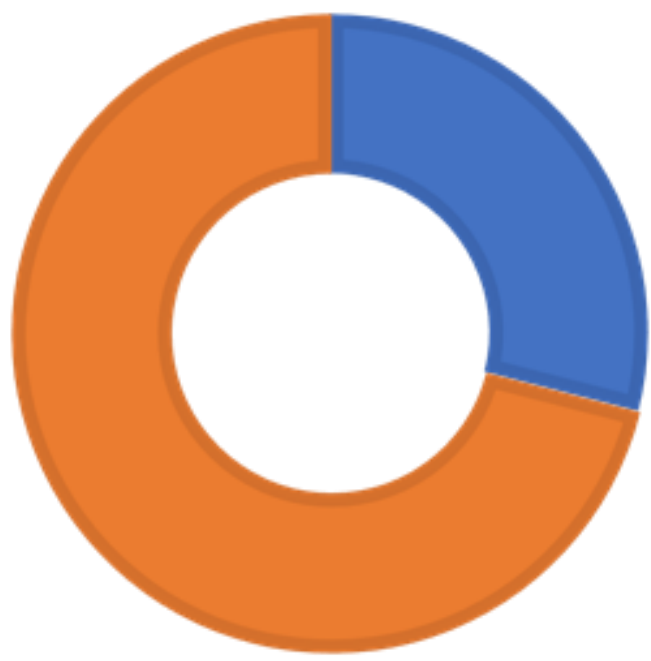




\section{Fig.6}

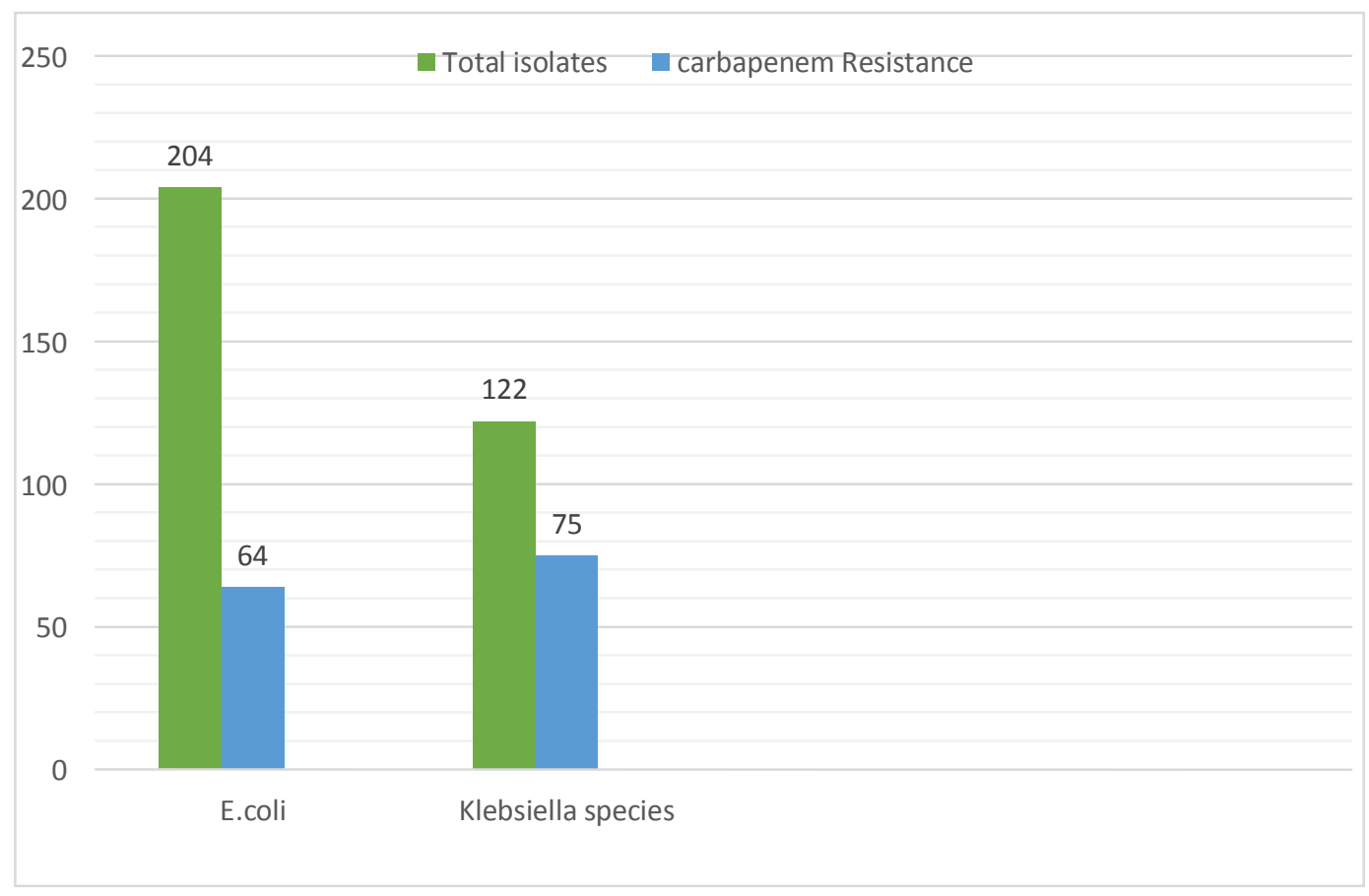

In our study, Escherichia coli was found to be more susceptible to Polymyxins, i.e, Colistin (100\%) and Polymyxins B (100\%), which correlates with the study done by Beatriz Suay et al., in 2019 where Colistin and Polymyxins were $100 \%$ sensitive to $E$. coli(18).

Klebsiella species were found to be highly sensitive to antibiotics Colistin (100\%) and Polymyxins (100\%), followed by Fosfomycin (59\%) whereas Cefuroxime (12.2\%) was found to be most resistant.

Similar findings were observed by Kathleen Chiotos et al., in their study - the highest sensitivity was observed to Polymyxins followed by Fosfomycin. (25)

The present study highlighted the burden of carbapenem resistance in critical and noncritical areas of our tertiary care hospital. In this study, out of $326(25.8 \%)$ strains of Escherichia coli and Klebsiella species, 139 (42.6\%) were carbapenem-resistant, 64 (46\%) isolates are of Escherichia coli and 75(53.9\%) isolates are of Klebsiella species.
Out of 326 strains of E. coli and Klebsiella isolated during the study period, 139 were resistant to imipenem. Out of these, in Escherichia coli 40 (28.7\%) and Klebsiella species $44(31.6 \%)$ were positive for MBL production by combined disk test (Imipenem + Imipenem EDTA). A similar result was observed in a study conducted by e André Birgy et al., in 2012 where 28.7\% were positive for MBL production by combined disk test. ${ }^{(11,18)}$

The concomitant presence of several different types of $\beta$-lactamases in resistant Enterobacteriaceae makes it more difficult to detect individual mechanisms, as one mechanism can mask another. Phenotypic detection of combined mechanisms of resistance, such as MBL-expressing isolates, is important for epidemiological purposes and for implementing rapid and specific infection control measures. This is a satisfactory and inexpensive method for characterizing the type of carbapenemase and for detecting associated resistance mechanisms in 
laboratories when PCR is not readily available. $^{(11)} \quad$ Carbapenem-resistant Enterobacteriaceae (CRE) pose an exponentially increasing threat to public health worldwide. These bacteria possess diverse and versatile mechanisms of drug resistance, which makes control and early detection of infections caused by CRE difficult. However, one striking feature noticed was the carbapenem resistance was more in the urine specimen in the case of both; Escherichia coli and Klebsiella species.

In the present study, we found that the Klebsiella species $(75 \%)$ and Escherichia coli $(64 \%)$ was the major reason of carbapenemase production but the organisms incidentally were susceptible to colistin and polymyxins (100\%) followed by other antibiotics. This is a welcome finding in times of increasing carbapenem resistance $\&$ is reason enough to suggest the use of Polymyxins in the health care settings where the carbapenems to be used for the treatment of infections caused by Enterobacteriaceae. Although outbreaks of carbapenemase-producing organisms have been difficult to control, there are data that if systematically implemented, rigorous infection control procedures can halt the spread of these organisms in both hospital and community-acquired infections.

\section{References}

1. Villegas M., Lolans K., Correa A., Suarez C., Lopez J., Vallejo M., et al., (2006) First detection of the plasmid-ediated class A carbapenemase KPC-2 inclinical isolates of Klebsiella pneumonia from South America. AntimicrobAgentsChemother50:28802882. (PMC free article) (PubMed) (GoogleScholar)

2. World Health Organization Antimicrobial Resistance: Global Report on Surveillance. WHO Press; Geneva,
Switzerland: 2014 ((accessed on 12 December 2017) :http://apps.who.int/iris/bitstream/10665/ 112642/1/9789241564748_eng.pdf.(Goo gle Scholar)

3. Blondeau $\mathbf{J}$ M. Current issues in the management of urinary tract infections: Extended- release ciprofloxacin as a novel treatment option. Drugs 2004;6: 611-28. (Links)

4. Falagas M E, Karageorgopoulos D E. Extended-spectrum beta-lactamaseproducing organisms. Journal of Hospital Infection 2009; 73: 345-54.(Links)

5. Hansen D S, Gottschau A, Kolmos H J.1998. Epidemiology of Klebsiella bacteraemia: a case control study using Escherichia coli bacteraemia as control. J Hosp Infect 38:119-132. doi:10.1016/S0195-6701(98)90065-2

Cross Ref PubMed Web of ScienceGoogleScholar)

6. Munoz-Price L S, Poirel L, Bonomo R A, et al., Clinical epidemiology of the global expansion of Klebsiella pneumonia carbapenemases. Lancet Infect Dis. 2013;13(9):785-96.I

7. Thomson K S. Extended-spectrum-betalactamase, AmpC, and Carbapenemase issues. J ClinMicrobiol. 2010;48: 1019 25. (PMC free article) (PubMed) (Google Scholar).

8. Mackie \& McCartney Practical Medical Microbiology, tests for identification of Enterobacteriaceae, $14^{\text {th }}$ edition by J. G Colle, A. G Fraser, B. PMarmion. A Simmons, P-361-364.

9. CLSI Guidance for control of infections with carbapenem-resistant or carbapenemase producing Enterobacteriaceae in acute care facilities CLSI home; (Online) CLSI Performance Standards for Antimicrobial Susceptibility Testing; 29th edition. CLSI SupplementM100.

10. Hemalatha V, Sekar U, Kamat V. 
Detection of metallo betalactamase producing Pseudomonas aeruginosa in hospitalized patients. IndianJMedRes.2005;122:148-52. (PubMed) (Google Scholar).

11. André Birgy, Philippe Bidet, Nathalie Genel, Catherine Doit, Dominique Decré, Guillaume Arlet, Edouard Bingen: Phenotypic Screening of Carbapenemases and Associated $\beta$-Lactamases in Carbapenem-Resistant

Enterobacteriaceae.

DOI: 10.1128/JCM.06131-11.

12. D A Pastel, Imipenem-cilastatin sodium, a broad-spectrum carbapenem antibiotic combination: 1986Sep;5(9):719-36.

13. Stewart, N. K.; et al., "Kinetic and structural requirements for carbapenemase activity in GES-type $\beta$ lactamases." Biochemistry 54.2 (2014):588-597.

14. bd bbl prompt inoculation system pkg INSERT \#5308-10 rev 06/2010 Bauer,a.w.,w.m.m.Kirby,j.c.Sherris,AND m.Turck.1966. Antibiotic Susceptibility Testing by a Standardized Single Disk Method. Am. J. Clin. Pathol. 45:493496.

15. FDA: US Food and Drug Administration (Internet). Silver Spring (MD): U.S. Department of Health and Human Services; - Combating Antibiotic Resistance; (updated 2018 Sep 10; cited 2019 Mar 31); (about 3 screens). Available from: https://www.fda.gov/ForConsumers/Cons umerUpdates/ucm092810.htm

16. Merck Manuals: Professional Version (Internet). Kenilworth (NJ): Merck \& Co., Inc.; c2017. Overview of Bacteria; (updated 2015 Jan; cited 2017 Mar 4); (about 2 screens). Available, from: http://www.merckmanuals.com/professio nal/infectious-diseases/bacteria-andantibacterial-drugs/overview-of-bacteria.

17. Sagar Aryal, Blood Agar- Composition, Preparation, Uses and Pictures.
MicrobiologyInfo.com. Last updated: October 26, 2018.

18. Beatriz Suay-García and María Teresa Pérez-Gracia* Present and Future of Carbapenem-resistant Enterobacteriaceae (CRE) Infections. Antibiotics (Basel). 2019 Sep; 8(3): 122. Published online 2019 Aug 19. doi: 10.3390/antibiotics8030122 PMCID:PMC6784177.

19. Kathleen Chiotos, M D,1,2,3 Jennifer $H$. Han, M D, MSCE,4,5,6 and Pranita D. Tamma, MD, MHS7. CarbapenemResistant Enterobacteriaceae Infections in Children. PMC 2017 Jan 1. Published in final edited form as: Curr Infect Dis Rep. 2016 Jan; 18(1): 2.doi:10.1007/s11908015-0510.

20. Wonkeun Song, S M. D., corresponding author1 Seong Geun Hong, M. D.,2 Dongeun Yong, M. D.,3 Seok Hoon Jeong, M. D.,3 HyunSooKim, M. D.,1 Han-SungKim, M. D.,1 Jae-Seok Kim, M.D.,1 and Il Kwon Bae, Ph.D.4 20. Combined Use of the Modified Hodge Test and Carbapenemase Inhibition Test for Detection of CarbapenemaseProducing Enterobacteriaceae and Metallo- $\beta$-Lactamase-Producing

Pseudomonas spp. Ann Lab Med.2015 Mar; 35(2): 212-219. Published online 2015 Feb 12. doi: 10.3343/alm. 2015.35.2.212.

21. Moritz Fritzenwanker, Dr.,1,2 Can Imirzalioglu, Dr.,1,2 Susanne Herold, Prof.,1,4 Florian M. Wagenlehner, Prof.,1,3 Klaus-Peter Zimmer, Prof.,1,5 and Trinad Chakraborty, Prof.1,2*Treatment Options for Carbapenem-Resistant Gram- Negative Infections Published online 2018 May 21. doi:10.3238/arztebl.2018.0345.

22. Latania K. Logan, Robert A. Weinstein The Epidemiology of CarbapenemResistant Enterobacteriaceae: The Impact and Evolution of a Global Menace. The 
Journal of Infectious Diseases, Volume 215, Issue suppl_1, 15 February 2017, Pages S28-S36, https://doi.org/10.1093/infdis/jiw282 Published:28March 2017.

23. Sakina Yousuf Ali and Taqdees Malik. Detection of carbapenem resistant Klebsiella species isolated from clinical samples. Pure and Applied Biology. Vol. 9, Issue 2, pp1662-1666.

24. Setegn Eshetie, Chandrashekhar Unakal, Aschalew Gelaw, Birhanu Ayelign, Mengistu Endris \& Feleke Moges. Multidrug resistant and carbapenemase producing Enterobacteriaceae among patients with urinary tract infection at referral Hospital, Northwest Ethiopia. Antimicrobial Resistance and Infection Controlvolume 4, Article number: 12(2015).

25. Cheng-Yen Kao,a Jing-Jou Yan,b Yu-
Chun Lin,c Po-Xing Zheng,c and JiunnJong Wu; Complete Genome Sequence of Carbapenem-Resistant Klebsiella pneumoniae Strain 1756, Isolated from a Pus Specimen Genome Announc. 2017 Mar; 5(13): e00066-17 Published online 2017 Mar 30. doi: 10.1128/genome A.00066-17.

26. Ling-bin Shu, $1 *$ Qun Lu,2* Ren-hua Sun,3 Le-qing Lin,4 Qiao-ling Sun,1 Jie Hu, 1 Hong-wei Zhou,1 Edward Wai-Chi Chan,5,6 Sheng Chen,5,6 and Rong Zhang;Prevalence and phenotypic characterization of carbapenem-resistant Klebsiella pneumoniae strains recovered from sputum and fecal samples of ICU patients in Zhejiang Province, China. Infect Drug Resist. 2019; 12:11-18. Published online 2018 Dec 18. doi: 10.2147/IDR.S175823.

\section{How to cite this article:}

Harvinder Kaur, Sneha Mohan, Tarana Sarwat and Dalip K. Kakru. 2021. Detection of Carbapenemase Production in Clinical Isolates of Escherichia Coli and Klebsiella Species. Int.J.Curr.Microbiol.App.Sci. 10(11): 438-450. doi: https://doi.org/10.20546/ijcmas.2021.1011.049 